\title{
硫酸催化的炔丙醇快速亲核取代反应
}

\author{
张顺吉* 刘会丽 \\ (淮北师范大学化学与材料科学学院 安徽淮北 235000)
}

\begin{abstract}
摘要 硫酸有效地催化炔丙醇和一系列碳和氧亲核试剂的直接亲核取代反应以形成碳-碳键和碳-氧键. 反应可以在未 除水溶剂中和空气条件下进行并获得良好的产率. 室温下大多数底物的反应能在 $1 \mathrm{~min}$ 内完成.

关键词 硫酸; 催化; 炔丙醇; 亲核取代
\end{abstract}

\section{Sulfuric Acid Catalyzed Rapid Nucleophilic Substitution of Propargyl Alcohols}

\author{
Zhang, Shunji* Liu, Huili \\ (Department of Chemistry, Huaibei Normal University, Huaibei, Anhui 235000)
}

\begin{abstract}
Sulfuric acid efficiently catalyzes the direct substitution of the hydroxyl group of propargylic alcohols with a variety of $\mathrm{C}$ - and $\mathrm{O}$-based nucleophiles to aid $\mathrm{C}-\mathrm{C}$ and $\mathrm{C}-\mathrm{O}$ bond formation. The reactions can be performed in an undried solvent under air atmosphere to obtain the desired products in good yields. In most cases, the reaction proceeds to completion in 1 min at room temperature.

Keywords sulfuric acid; catalyzed; propargyl alcohols; nucleophilic substitution
\end{abstract}

\section{Introduction}

The propargyl alcohol framework is a versatile structural motif frequently found in natural products, pharmaceutical intermediates, and synthetic compounds. Modification of the propargyl alcohol structure is a significant research topic. Propargyl alcohols are readily prepared by conventional addition reaction between the corresponding acetylides and carbonyl compounds, and have been employed as starting materials in various transformations. ${ }^{[1]}$

Nucleophilic substitution of the hydroxyl group of propargyl alcohols is widely investigated in organic synthesis toward carbon-carbon and carbon-heteroatom bond formation. Recently, methods for the direct substitution of propargylic alcohols using gold, ${ }^{[2]}$ cobalt, ${ }^{[3]}$ rhenium, ${ }^{[4]}$ and ruthenium, ${ }^{[5]}$ metal chlorides, ${ }^{[6]}$ and triflates ${ }^{[7]}$ such as $\mathrm{Yb}(\mathrm{OTf})_{3}$ and $\mathrm{Al}(\mathrm{OTf})_{3}$ have been reported. However, the widespread application of these methods is limited due to the high cost of the catalysts, as well as the need for anhydrous conditions and elevated temperatures. The development of propargylation reactions using simple air- and moisture-stable catalysts is highly desired. The use of Brønsted acids in the direct substitution of propargyl alcohols is possible solution to this problem as this strategy is more environmentally friendly compared to the existing protocols. Based on this concept, several catalytic methodologies for the substitution of propargyl alcohols in the presence of Brønsted acid catalysts such as $p$-toluenesulfonic acid (PTSA) ${ }^{[8]} \mathrm{HBF}_{4}{ }^{[9]}$ 4-nitrobenzenesulfonic acid, $^{[10]}$ trifluoroacetic acid, ${ }^{[11]}$ trifluoromethanesulfonic acid, ${ }^{[12]}$ perchloric acid, ${ }^{[13]}$ phosphomolybdic acid, ${ }^{[14]}$ zeolite, ${ }^{[15]}$ Amberlyst-15, ${ }^{[16]}$ and Amberlite IR-120H resin ${ }^{[17]}$ have been reported. However, there is no established method for sulfuric acid-catalyzed substitution of the hydroxyl group of propargyl alcohols with nucleophiles toward $\mathrm{C}-\mathrm{C}$ and $\mathrm{C}-\mathrm{O}$ bond formation. Here we report our results for the sulfuric acid-catalyzed metal-free direct nucleophilic substitution of the hydroxyl group of propargyl alcohols with various nucleophiles at room temperature to afford the corresponding products in good yields (Scheme 1). This method does not require expensive catalysts, explosive solvents, heating or a long reaction time. Furthermore, the reactions can be performed under mild conditions without the need for precautions to exclude moisture or air from the reaction system. In most cases, the reaction proceeds to completion within $1 \mathrm{~min}$.

\footnotetext{
* Corresponding author. E-mail: sjzhang@chnu.edu.cn

Received November 27, 2019; revised January 6, 2020; published online January 21, 2020.
} 


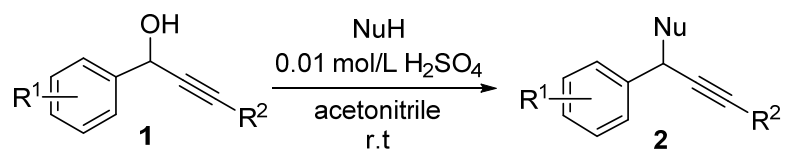

$\mathrm{R}^{1}=\mathrm{MeO}, \mathrm{Me}, \mathrm{BnO}, \mathrm{Cl}, \mathrm{CF}_{3} ; \mathrm{R}^{2}=$ aryl, alkyl

$\mathrm{NuH}=$ nucleophile

Scheme 1 Nucleophilic substitution of propargyl alcohols

\section{Results and discussion}

First the sulfuric acid-catalyzed nucleophilic substitution of 1-(3,4-dimethoxyphenyl)-3-phenyl-2-propyn-1-ol (1a) was investigated with phenol as the nucleophile (Table 1, Entry 1). Gratifyingly, the reaction performed using 0.01 $\mathrm{mol} / \mathrm{L}$ sulfuric acid in acetonitrile at room temperature afforded the corresponding C-nucleophilic substitution product 2a, which was isolated and characterized in good yield. Aiming to extend the reaction to other nucleophilic reagents, 1a was treated with diverse nucleophiles such as anisole, furan, 6-chloroindole, and 6-nitroindole. The starting material was completely consumed within $1 \mathrm{~min}$, which confirmed by thin-layer chromatography (TLC), and the corresponding products were isolated in good yields (Table 1, Entries 2 5).

To determine the effectiveness of the catalyst, the reactions were conducted with different concentrations of sulfuric acid $(0.005,0.01,0.05,0.10 \mathrm{~mol} / \mathrm{L})$ in acetonitrile. It was determined that $0.01 \mathrm{~mol} / \mathrm{L}$ sulfuric acid was found to be the optimal concentration, and a lower concentration of the acid necessitated a longer reaction time. Although the reactions were run in acetonitrile, they also worked well in dichloromethane, toluene, and tetrahydrofuran.

Next, the scope and generality of the reaction were investigated by varying the substituents, $R^{1}$ and $R^{2}$, on the propargyl alcohols and found that the reaction proceeded successfully to furnish the expected products (Table 1, Entries $6 \sim 20$ ). Substrates with electron-withdrawing groups on the aromatic rings underwent the reaction much more slowly (over several hours) than did those with electron-donating groups in the previous catalytic system; ${ }^{[1]}$ nonetheless, the sulfuric acid-catalyzed nucleophilic substitution of propargyl alcohols could be completed $1 \mathrm{~min}$ in most cases, indicating that sulfuric acid serves as an effective catalyst for the aforementioned reaction. With all the nucleophiles tested, substitution resulted in a regioselective attack by the aromatic carbon with the highest electron density, that is, C-2 for furans, C-3 for indoles, and C-4 for phenols. $^{[10]}$

Next, for extending the reaction to O-nucleophilic reagents, 1,3-diarylpropargyl alcohols were treated with alcohols such as methanol, ethanol, isopropanol, and cinnamyl alcohol. The starting material was completely consumed within $1 \mathrm{~min}$ and afforded a complex inseparable mixture, as revealed by thin-layer chromatography (TLC). However when the terminal aromatic ring on the alkyne was replaced with aliphatic group as in $\mathbf{1 g}$ or $\mathbf{1 h}$, only a single spot was observed on the TLC spot. After work-up, the corresponding O-substituted products were isolated in excellent yield (Table 1, Entries 21 25).

Based on previous studies, ${ }^{[1 \mathrm{~b}]}$ it is assumed that the reaction proceeds by direct substitution of the hydroxyl group with the nucleophile via $\mathrm{S}_{\mathrm{N}} 1$-type mechanism. First, the hydroxyl group of propargyl alcohol is protonated by sulfuric acid, and then, water is eliminated to generate a propargyl carbocation. The nucleophile attacks the propargyl carbocation and is immediately deprotonated to afford the corresponding product. The eliminated proton is captured by a sulfate anion to regenerate sulfuric acid.

\section{Conclusions}

A simple strategy for the direct nucleophilic substitution of the hydroxyl group of propargyl alcohols toward the construction of $\mathrm{C}-\mathrm{C}$ and $\mathrm{C}-\mathrm{O}$ bonds catalyzed by an effective protonic acid and sulfuric acid has been developed. The reaction proceeds effectively under mild conditions (room temperature, short time, in undried solvent, under air) in the presence of a simple, and commercially available catalyst.

\section{Experimental}

\subsection{General information}

All chemicals and solvents were used without further purification as commercially available. All manipulations were carried out under air atmosphere. Flash column chromatography was generally conducted on silica gel (200 300 mesh) and reactions were monitored by thin layer chromatography (TLC) using UV light to visualize the course of reactions. ${ }^{1} \mathrm{H}$ NMR and ${ }^{13} \mathrm{C}$ NMR spectra were measured on a Bruker spectrometer using $\mathrm{CDCl}_{3}$ or DMSO- $d_{6}$ as solvent. ${ }^{1} \mathrm{H}$ NMR spectra was recorded with TMS $(\delta 0.00)$ as internal reference. ${ }^{13} \mathrm{C}$ NMR spectra was recorded with $\mathrm{CDCl}_{3}\left(\delta\right.$ 77.0) or DMSO- $d_{6}(\delta$ 39.5) as internal reference. HRMS were performed on a Agilent ESI-quad- rupole. The melting points were obtained on a Laboratory Devices WRS-1B melting apparatus and were uncorrected. 1,3-Disubstituted propargyl alcohols (1) were prepared according to the literature. ${ }^{[1 \mathrm{a}]}$

4.2 Typical procedure for the preparation of 1,3disubstituted propargyl derivatives (2)

$0.01 \mathrm{~mol} / \mathrm{L} \mathrm{H}_{2} \mathrm{SO}_{4} / \mathrm{MeCN}$ solution was prepared by dissolving $0.1 \mathrm{~mL}$ sulphuric acid in $180 \mathrm{~mL}$ acetonitrile.

Propargyl alcohol $1(0.2 \mathrm{mmol})$ and nucleophile $(0.24$ mmol, 1.2 equiv.) were added to the solution of $0.01 \mathrm{~mol} / \mathrm{L}$ $\mathrm{H}_{2} \mathrm{SO}_{4} / \mathrm{MeCN}(2 \mathrm{~mL})$ at room temperature. The reaction mixture was stirred at the same temperature and the progress of the reaction was monitored by thin layer chromatography (TLC). The reaction mixture was quenched by $10 \%$ aq. $\mathrm{Na}_{2} \mathrm{CO}_{3}$ solution, and extracted with ethyl acetate. The combined organic phase was washed by brine $(20 \mathrm{~mL})$, dried by $\mathrm{Na}_{2} \mathrm{SO}_{4}$, filtrated and concentrated in vacuum. The crude material was purified by flash column chromatography (silica gel, PE-EtOAc) to obtain the desired product 2. 
Table 1 Nucleophilic substitution of propargyl alcohols with nucleophiles ${ }^{a}$

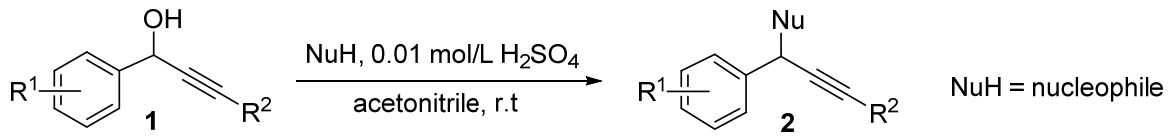

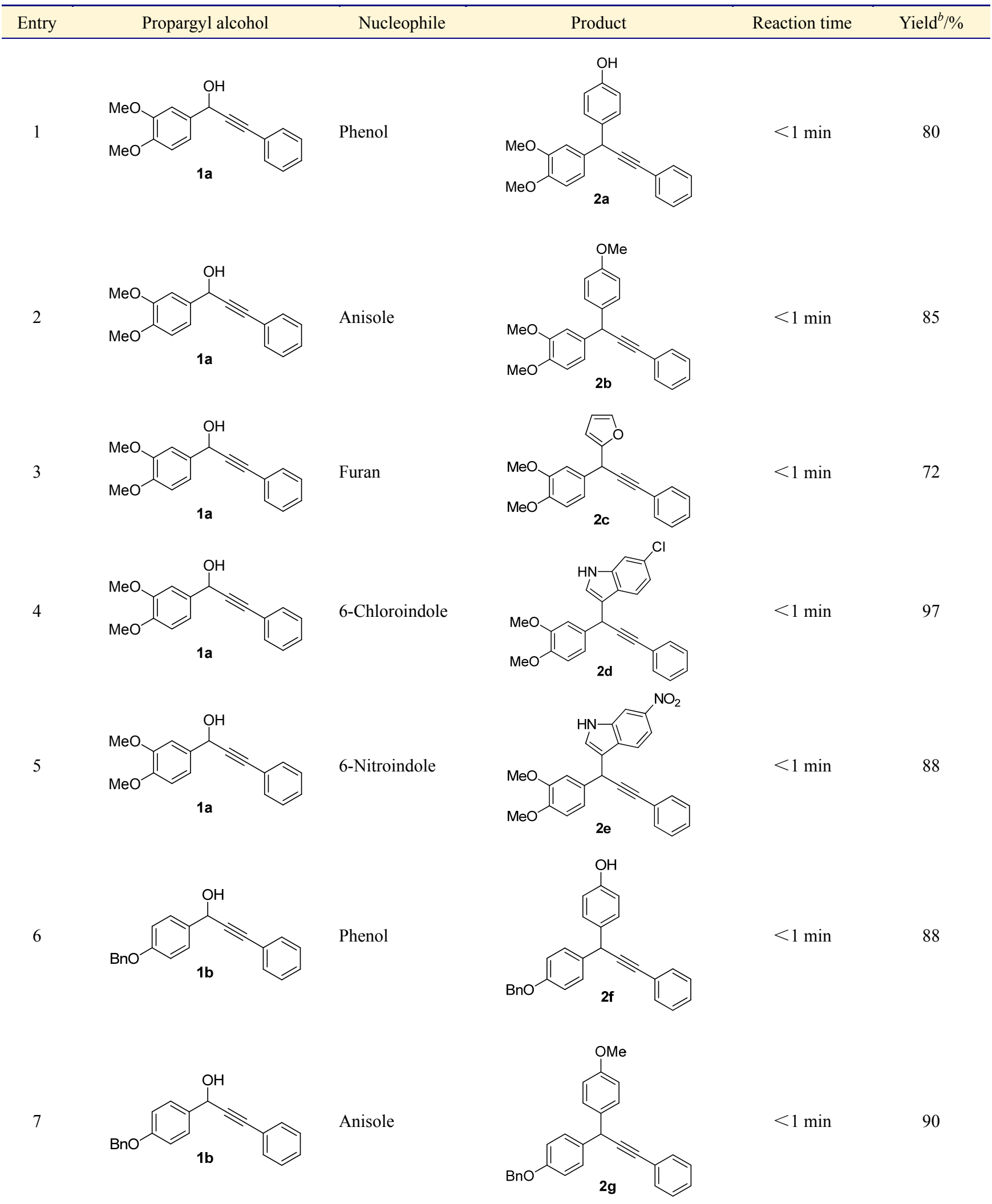




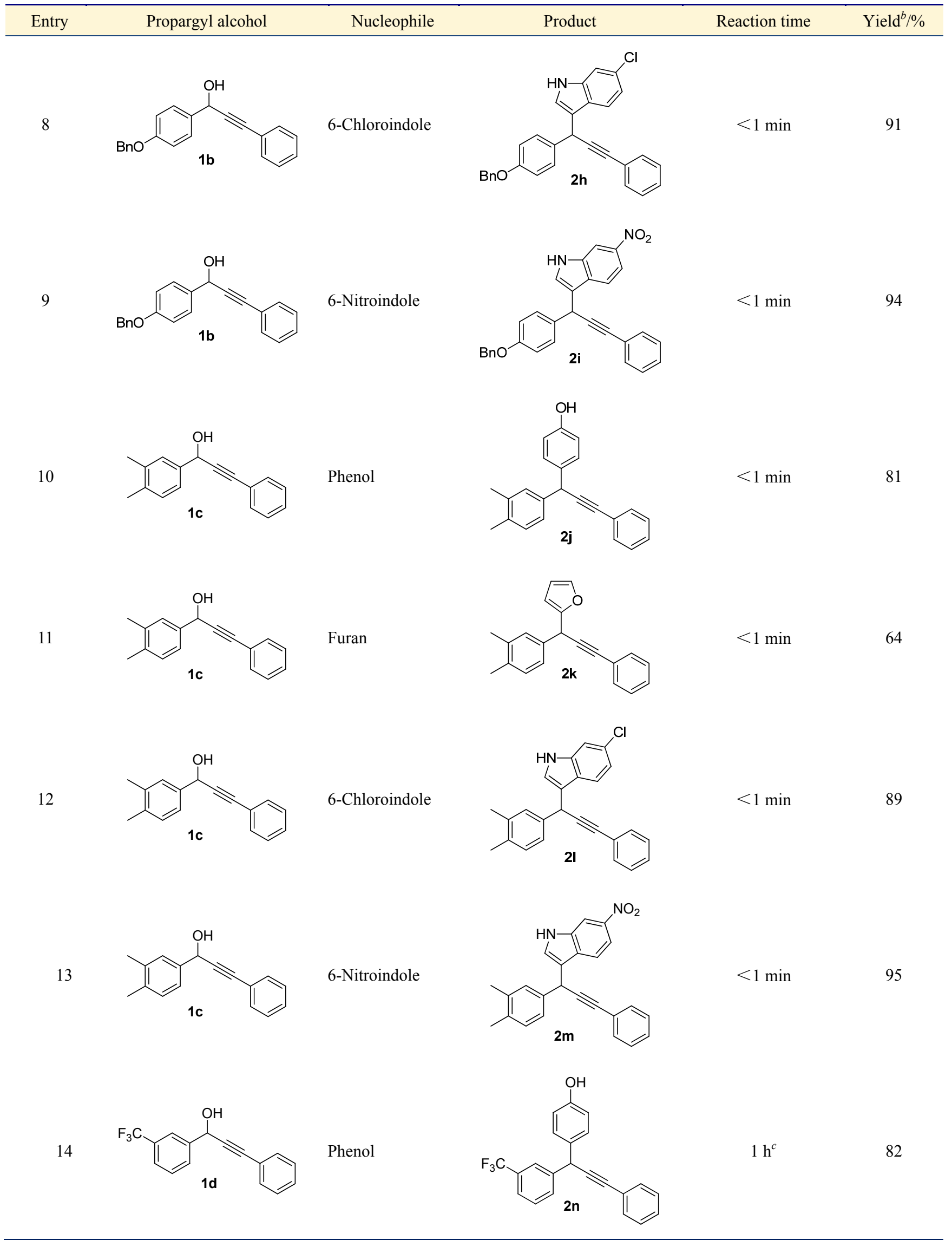




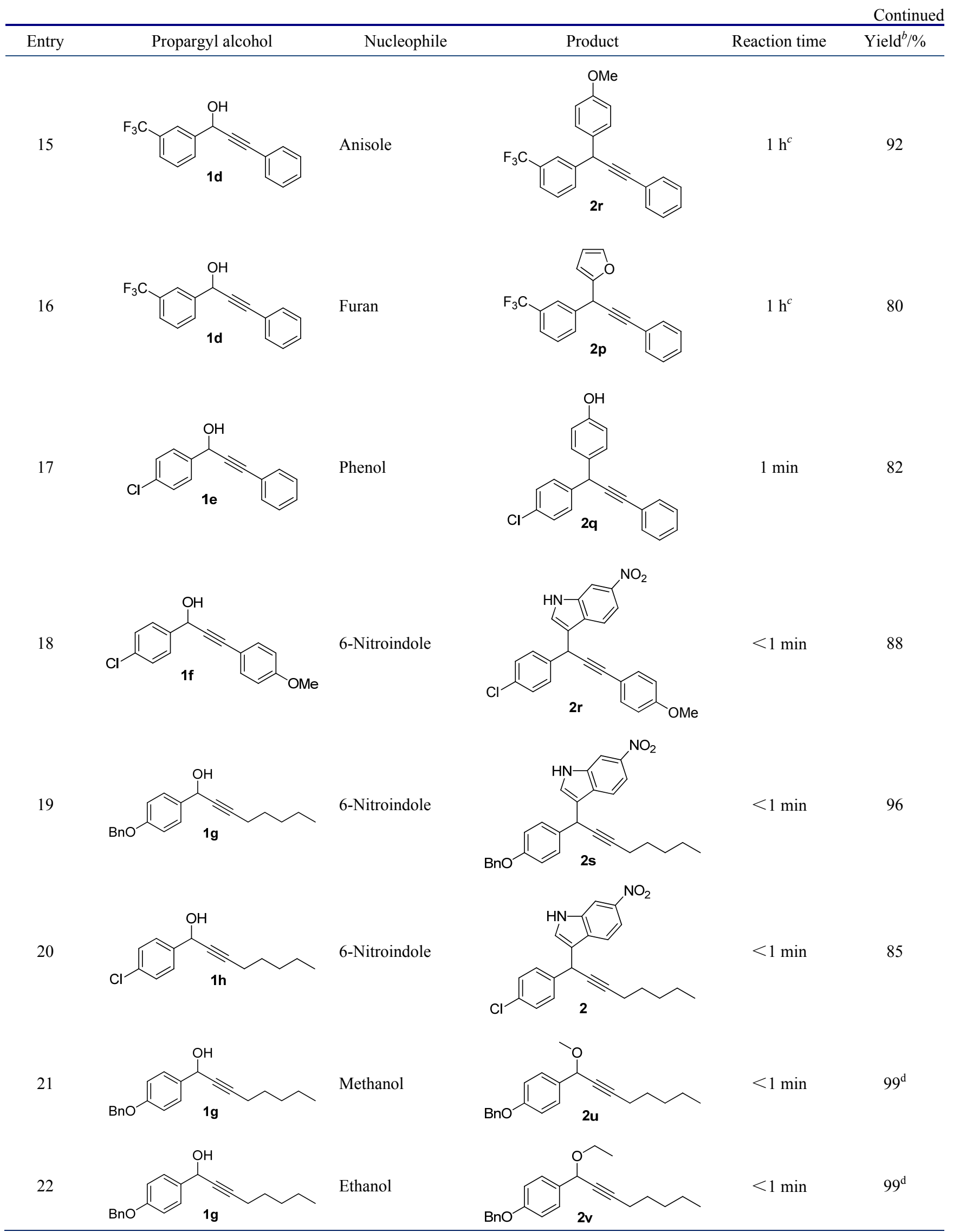


Continued

Entry Propargyl alcohol Reaction time $\mathrm{Yield}^{b} / \%$

${ }^{a}$ Reaction conditions: propargyl alcohol $(0.2 \mathrm{mmol})$, nucleophile $(0.24 \mathrm{mmol}), 0.01 \mathrm{~mol} / \mathrm{L} \mathrm{H}_{2} \mathrm{SO}_{4} /$ acetonitrile solution $(2 \mathrm{~mL}) .{ }^{b}$ Isolated yield. ${ }^{c}$ It will take $20 \mathrm{~h}$ to complete the reaction if $0.01 \mathrm{~mol} / \mathrm{L} \mathrm{H}_{2} \mathrm{SO}_{4}$ is replaced by $10 \mathrm{~mol} \%$ PTSA. ${ }^{d}$ Reaction conditions: propargyl alcohol ( $\left.0.2 \mathrm{mmol}\right)$, nucleophile (1.0 mmol), $0.01 \mathrm{~mol} / \mathrm{L}$ $\mathrm{H}_{2} \mathrm{SO}_{4}$ /acetonitrile solution $(2 \mathrm{~mL})$.

4-(1-(3,4-Dimethoxyphenyl)-3-phenylprop-2-yn-1-yl)phenol (2a): Yield 80\%, light yellow oil. ${ }^{1} \mathrm{H}$ NMR (600 $\left.\mathrm{MHz}, \mathrm{CDCl}_{3}\right) \delta: 7.48 \sim 7.44(\mathrm{~m}, 2 \mathrm{H}), 7.32 \sim 7.26(\mathrm{~m}, 5 \mathrm{H})$, $6.98 \sim 6.93(\mathrm{~m}, 2 \mathrm{H}), 6.82(\mathrm{~d}, J=7.8 \mathrm{~Hz}, 1 \mathrm{H}), 6.79(\mathrm{~d}, J=$ $8.4 \mathrm{~Hz}, 2 \mathrm{H}), 5.10$ (s, 1H), 3.85 (s, 3H), $3.84(\mathrm{~s}, 3 \mathrm{H}) ;{ }^{13} \mathrm{C}$ NMR $\left(150 \mathrm{MHz}, \mathrm{CDCl}_{3}\right) \delta: 154.6,149.0,147.9,134.7$, $134.1,131.7,129.0,128.3,128.0,123.6,120.0,115.4$, $111.3,111.2,90.7,84.7,56.0,55.9,42.5$; IR (KBr) v: 3432, 3059, 3006, 2958, 2937, 1597, 1511, 1445, 1264, 1234 $\mathrm{cm}^{-1}$; HRMS (ESI) calcd for $\mathrm{C}_{23} \mathrm{H}_{21} \mathrm{O}_{3}[\mathrm{M}+\mathrm{H}]^{+}$ 345.1485 , found 345.1484 .

1,2-Dimethoxy-4-(1-(4-methoxyphenyl)-3-phenylprop2-yn-1-yl)benzene (2b): Yield 85\%, colorless oil. ${ }^{1} \mathrm{H}$ NMR $\left(600 \mathrm{MHz}, \mathrm{CDCl}_{3}\right) \delta: 7.48 \sim 7.45(\mathrm{~m}, 2 \mathrm{H}), 7.34(\mathrm{~d}, J=8.4$ $\mathrm{Hz}, 2 \mathrm{H}), 7.32 \sim 7.28(\mathrm{~m}, 3 \mathrm{H}), 6.98 \sim 6.94(\mathrm{~m}, 2 \mathrm{H}), 6.86(\mathrm{~d}$, $J=8.4 \mathrm{~Hz}, 2 \mathrm{H}), 6.82(\mathrm{~d}, J=8.4 \mathrm{~Hz}, 1 \mathrm{H}), 5.12(\mathrm{~s}, 1 \mathrm{H}), 3.86$ $(\mathrm{s}, 3 \mathrm{H}), 3.85(\mathrm{~s}, 3 \mathrm{H}), 3.79(\mathrm{~s}, 3 \mathrm{H}) ;{ }^{13} \mathrm{C} \mathrm{NMR}(150 \mathrm{MHz}$, $\left.\mathrm{CDCl}_{3}\right) \delta: 158.5,149.0,148.0,134.7,134.1,131.7,128.8$, 128.3, 128.0, 123.6, 119.9, 114.0, 111.2, 111.2, 90.7, 84.6, 55.94, 55.89, 55.3, 42.5; IR (KBr) v: 3060, 3002, 2956, 2935, 1599, 1511, 1460, 1256, $1175 \mathrm{~cm}^{-1}$; HRMS (ESI) calcd for $\mathrm{C}_{24} \mathrm{H}_{23} \mathrm{O}_{3}[\mathrm{M}+\mathrm{H}]^{+}$359.1642, found 359.1636.

2-(1-(3,4-Dimethoxyphenyl)-3-phenylprop-2-yn-1-yl)furan (2c): Yield $72 \%$, light yellow oil. ${ }^{1} \mathrm{H}$ NMR $(600 \mathrm{MHz}$, $\left.\mathrm{CDCl}_{3}\right) \delta: 7.59(\mathrm{~s}, 1 \mathrm{H}), 7.50 \sim 7.46(\mathrm{~m}, 2 \mathrm{H}), 7.40 \sim 7.37(\mathrm{~m}$, $3 \mathrm{H}), 7.04(\mathrm{~s}, 1 \mathrm{H}), 6.97(\mathrm{dd}, J=7.8,18.6 \mathrm{~Hz}, 1 \mathrm{H}), 6.41(\mathrm{~s}$, $1 \mathrm{H}), 6.31(\mathrm{~s}, 1 \mathrm{H}), 5.47(\mathrm{~s}, 1 \mathrm{H}), 3.74(\mathrm{~s}, 6 \mathrm{H}) ;{ }^{13} \mathrm{C}$ NMR $(150$ $\left.\mathrm{MHz}, \mathrm{CDCl}_{3}\right) \delta: 154.0,149.2,148.6,143.1,131.9,131.7$, 129.2, 129.0, 122.8, 120.2, 112.5, 112.0, 111.0, 106.9, 88.9, $83.5,56.1,56.0,36.6$; IR (KBr) v: 3059, 3001, 2957, 2936, 2836, 1597, 1513, 1460, 1264, $1179 \mathrm{~cm}^{-1}$; HRMS (ESI) calcd for $\mathrm{C}_{21} \mathrm{H}_{19} \mathrm{O}_{3}[\mathrm{M}+\mathrm{H}]^{+}$319.1329, found 319.1327 .

6-Chloro-3-(1-(3,4-dimethoxyphenyl)-3-phenylprop-2yn-1-yl)-1H-indole (2d): Yield 97\%. Yellow solid, m.p. $167 \sim 168{ }^{\circ} \mathrm{C} ;{ }^{1} \mathrm{H} \mathrm{NMR}\left(600 \mathrm{MHz}, \mathrm{CDCl}_{3}\right) \delta$ : $8.22(\mathrm{~s}, 1 \mathrm{H})$, $7.51(\mathrm{~d}, J=8.4 \mathrm{~Hz}, 1 \mathrm{H}), 7.46 \sim 7.42(\mathrm{~m}, 2 \mathrm{H}), 7.31(\mathrm{~s}, 1 \mathrm{H})$, $7.30 \sim 7.26(\mathrm{~m}, 3 \mathrm{H}), 7.08(\mathrm{~s}, 1 \mathrm{H}), 7.06 \sim 7.01(\mathrm{~m}, 3 \mathrm{H}), 6.82$ $(\mathrm{d}, J=7.8 \mathrm{~Hz}, 1 \mathrm{H}), 5.37(\mathrm{~s}, 1 \mathrm{H}), 3.85(\mathrm{~s}, 3 \mathrm{H}), 3.82(\mathrm{~s}, 3 \mathrm{H})$; ${ }^{13} \mathrm{C}$ NMR $\left(150 \mathrm{MHz}, \mathrm{CDCl}_{3}\right) \delta: 149.0,148.0,137.2,133.6$, $131.7,128.3,128.2,128.0,124.7,123.6,123.3,120.6$, $120.3,120.0,117.2,111.3,111.2,111.1,90.4,83.5,56.0$, 55.9, 35.1; IR (KBr) v: 2415, 3354, 3060, 3000, 2958, 2936, 2836, 1597, 1512, 1457, 1262, $1139 \mathrm{~cm}^{-1}$; HRMS (ESI) calcd for $\mathrm{C}_{25} \mathrm{H}_{20} \mathrm{ClNNaO}_{2}[\mathrm{M}+\mathrm{Na}]^{+}$424.1075, found 424.1079 .

3-(1-(3,4-Dimethoxyphenyl)-3-phenylprop-2-yn-1-yl)-6nitro- $1 H$-indole (2e): Yield $88 \%$. Yellow solid, m.p. $>$ $197{ }^{\circ} \mathrm{C}$ (dec.); ${ }^{1} \mathrm{H}$ NMR (600 MHz, $\left.\mathrm{CDCl}_{3}\right) \delta: 8.63$ (s, 1H), $8.34(\mathrm{~s}, 1 \mathrm{H}), 7.98$ (d, $J=8.4 \mathrm{~Hz}, 1 \mathrm{H}), 7.68$ (d, $J=8.4 \mathrm{~Hz}$, $1 \mathrm{H}), 7.45(\mathrm{~d}, J=3.0 \mathrm{~Hz}, 2 \mathrm{H}), 7.41(\mathrm{~s}, 1 \mathrm{H}), 7.31(\mathrm{~m}, 3 \mathrm{H})$, 7.05 (s, 2H), $6.85(\mathrm{~d}, J=6.0 \mathrm{~Hz}, 1 \mathrm{H}), 5.42(\mathrm{~s}, 1 \mathrm{H}), 3.88$ (s, $1 \mathrm{H}), 3.85(\mathrm{~s}, 1 \mathrm{H}) ;{ }^{13} \mathrm{C} \mathrm{NMR}\left(150 \mathrm{MHz}, \mathrm{CDCl}_{3}\right) \delta: 149.2$, $148.3,143.6,135.3,132.9,131.7,130.7,128.3,128.2$, 128.1, 123.3, 120.0, 119.7, 118.4, 115.2, 111.2, 108.2, 89.7, 83.9, 56.0, 34.9; IR (KBr) v: 3375, 2362, 1607, 1512, 1457, $1340,1230,1136 \mathrm{~cm}^{-1}$; HRMS (ESI) calcd for $\mathrm{C}_{25} \mathrm{H}_{21} \mathrm{~N}_{2} \mathrm{O}_{4}$ $[\mathrm{M}+\mathrm{H}]^{+}$413.1496, found 413.1495.

4-(1-(4-(Benzyloxy)phenyl)-3-phenylprop-2-yn-1-yl)phenol (2f): Yield 88\%, light yellow oil. ${ }^{1} \mathrm{H}$ NMR $(600$ $\left.\mathrm{MHz}, \mathrm{CDCl}_{3}\right) \delta: 7.47 \sim 7.44(\mathrm{~m}, 2 \mathrm{H}), 7.41(\mathrm{~d}, J=7.2 \mathrm{~Hz}$, $2 \mathrm{H}), 7.38 \sim 7.34(\mathrm{~m}, 2 \mathrm{H}), 7.33 \sim 7.26(\mathrm{~m}, 8 \mathrm{H}), 6.92(\mathrm{~d}, J=$ $9.0 \mathrm{~Hz}, 2 \mathrm{H}), 6.76(\mathrm{~d}, J=8.4 \mathrm{~Hz}, 2 \mathrm{H}), 5.10(\mathrm{~s}, 1 \mathrm{H}), 5.03(\mathrm{~s}$, $2 \mathrm{H}) ;{ }^{13} \mathrm{C}$ NMR $\left(150 \mathrm{MHz}, \mathrm{CDCl}_{3}\right) \delta: 157.8,154.5,137.1$, $134.6,134.4,131.7,129.1,128.9,128.6,128.3,128.0$, $127.9,127.5,123.6,115.5,115.0,90.8,84.6,70.1,42.1$; IR (KBr) v: 3397, 3061, 3032, 2930, 2869, 1606, 1508, 1448, $1241,1174 \mathrm{~cm}^{-1}$; HRMS (ESI) calcd for $\mathrm{C}_{28} \mathrm{H}_{22} \mathrm{NaO}_{2}[\mathrm{M}+$ $\mathrm{Na}]^{+}$413.1512, found 413.1516.

1-(Benzyloxy)-4-(1-(4-methoxyphenyl)-3-phenylprop-2yn-1-yl)benzene (2g): Yield 90\%, colorless oil. ${ }^{1} \mathrm{H}$ NMR 
$\left(600 \mathrm{MHz}, \mathrm{CDCl}_{3}\right) \delta: 7.48 \sim 7.45(\mathrm{~m}, 2 \mathrm{H}), 7.42(\mathrm{~d}, J=7.8$ $\mathrm{Hz}, 2 \mathrm{H}), 7.39 \sim 7.35(\mathrm{~m}, 2 \mathrm{H}), 7.35 \sim 7.27(\mathrm{~m}, 8 \mathrm{H}), 6.93(\mathrm{~d}$, $J=8.4 \mathrm{~Hz}, 2 \mathrm{H}), 6.85$ (d, $J=8.4 \mathrm{~Hz}, 2 \mathrm{H}), 5.04$ (s, 2H), 3.78 $(\mathrm{s}, 3 \mathrm{H}) ;{ }^{13} \mathrm{C} \mathrm{NMR}\left(150 \mathrm{MHz}, \mathrm{CDCl}_{3}\right) \delta: 158.5,157.7,137.1$, $134.6,134.3,132.3,131.7,128.9,128.6,128.2,128.0$, $127.9,127.5,123.6,114.9,114.0,90.8,84.6,70.1,55.3$, 42.1; IR (KBr) v: 3032, 2932, 2836, 1606, 1508, 1459, $1248,1176 \mathrm{~cm}^{-1}$; HRMS (ESI) calcd for $\mathrm{C}_{28} \mathrm{H}_{22} \mathrm{NaO}_{2}[\mathrm{M}+$ $\mathrm{H}]^{+}$405.1849, found 405.1836 .

3-(1-(4-(Benzyloxy)phenyl)-3-phenylprop-2-yn-1-yl)-6chloro- $1 H$-indole $(\mathbf{2 h})$ : Yield 91\%. White solid, m.p. $137 \sim$ $138{ }^{\circ} \mathrm{C} ;{ }^{1} \mathrm{H} \mathrm{NMR}\left(600 \mathrm{MHz}, \mathrm{CDCl}_{3}\right) \delta: 11.15(\mathrm{~d}, J=1.8 \mathrm{~Hz}$, $1 \mathrm{H}), 7.53(\mathrm{~d}, J=9.0 \mathrm{~Hz}, 1 \mathrm{H}), 7.49 \sim 7.34(\mathrm{~m}, 13 \mathrm{H}), 7.31(\mathrm{t}$, $J=7.2 \mathrm{~Hz}, 1 \mathrm{H}), 7.00 \sim 6.96(\mathrm{~m}, 3 \mathrm{H}), 5.54(\mathrm{~s}, 1 \mathrm{H}), 5.06(\mathrm{~s}$, $2 \mathrm{H}) ;{ }^{13} \mathrm{C}$ NMR $\left(150 \mathrm{MHz}, \mathrm{CDCl}_{3}\right) \delta: 157.6,137.6,134.2$, $131.8,129.1,129.0,128.9,128.7,128.3,128.1,126.5$, 124.8, 124.7, 123.4, 120.8, 119.4, 116.2, 115.2, 111.8, 91.7, 83.1, 69.7, 34.1; IR (KBr) v: 3425, 3062, 3034, 2930, 2868, $1608,1507,1451,1242,1175 \mathrm{~cm}^{-1}$; HRMS (ESI) calcd for $\mathrm{C}_{30} \mathrm{H}_{23} \mathrm{CINO}[\mathrm{M}+\mathrm{H}]^{+}$448.1463, found 448.1451.

3-(1-(4-(Benzyloxy)phenyl)-3-phenylprop-2-yn-1-yl)-6nitro- $1 H$-indole (2i): Yield 94\%. Yellow solid, m.p. $>$ $140{ }^{\circ} \mathrm{C}$ (dec.); ${ }^{1} \mathrm{H}$ NMR (600 MHz, $\left.\mathrm{CDCl}_{3}\right) \delta: 8.48$ (s, 1H), $8.33(\mathrm{~s}, 1 \mathrm{H}), 7.97(\mathrm{~d}, J=8.4 \mathrm{~Hz}, 1 \mathrm{H}), 7.65(\mathrm{~d}, J=8.4 \mathrm{~Hz}$, $1 \mathrm{H}), 7.50 \sim 7.24(\mathrm{~m}, 13 \mathrm{H}), 6.96(\mathrm{~d}, J=7.2 \mathrm{~Hz}, 1 \mathrm{H}), 5.42(\mathrm{~s}$, 1H), $5.05(\mathrm{~s}, 2 \mathrm{H}) ;{ }^{13} \mathrm{C}$ NMR $\left(150 \mathrm{MHz}, \mathrm{CDCl}_{3}\right) \delta: 158.0$, $143.6,137.0,135.3,132.8,131.7,130.7,128.9,128.6$, $128.3,128.2,128.1,128.0,127.5,123.3,119.7,118.6$, $115.2,115.1,108.2,89.7,83.8,70.1,34.5$; IR (KBr) $v$ : 3344, 2959, 2928, 2857, 1610, 1508, 1456, 1323, 1244, $1172 \mathrm{~cm}^{-1}$; HRMS (ESI) calcd for $\mathrm{C}_{30} \mathrm{H}_{23} \mathrm{~N}_{2} \mathrm{O}_{3}[\mathrm{M}+\mathrm{H}]^{+}$ 459.1703, found 459.1704.

4-(1-(3,4-Dimethylphenyl)-3-phenylprop-2-yn-1-yl)phenol (2j): Yield 81\%, light yellow oil. ${ }^{1} \mathrm{H}$ NMR (600 $\left.\mathrm{MHz}, \mathrm{CDCl}_{3}\right) \delta: 7.48 \sim 7.44(\mathrm{~m}, 2 \mathrm{H}), 7.32 \sim 7.27(\mathrm{~m}, 5 \mathrm{H})$, $7.16(\mathrm{~s}, 1 \mathrm{H}), 7.14(\mathrm{~d}, J=7.8 \mathrm{~Hz}, 1 \mathrm{H}), 7.08(\mathrm{~d}, J=7.8 \mathrm{~Hz}$, $1 \mathrm{H}), 6.77(\mathrm{~d}, J=8.4 \mathrm{~Hz}, 1 \mathrm{H}), 5.09(\mathrm{~s}, 1 \mathrm{H}), 2.23(\mathrm{~s}, 3 \mathrm{H}), 2.22$ $(\mathrm{s}, 3 \mathrm{H}) ;{ }^{13} \mathrm{C} \mathrm{NMR}\left(150 \mathrm{MHz}, \mathrm{CDCl}_{3}\right) \delta: 154.4,139.5,136.8$, $135.1,134.4,131.7,129.9,129.1,128.2,127.9,125.2$, 123.7, 115.4, 90.8, 84.5, 42.5, 19.9, 19.4; IR (KBr) v: 3378, 3054, 3022, 2922, 1603, 1508, 1446, 1265, $1172 \mathrm{~cm}^{-1}$; HRMS (ESI) calcd for $\mathrm{C}_{23} \mathrm{H}_{21} \mathrm{O}[\mathrm{M}+\mathrm{H}]^{+} 313.1587$, found 313.1587 .

2-(1-(3,4-Dimethylphenyl)-3-phenylprop-2-yn-1-yl)furan (2k): Yield 64\%, colorless oil. ${ }^{1} \mathrm{H}$ NMR $(600 \mathrm{MHz}$, $\left.\mathrm{CDCl}_{3}\right) \delta: 7.48 \sim 7.45(\mathrm{~m}, 2 \mathrm{H}), 7.33(\mathrm{~s}, 1 \mathrm{H}), 7.30 \sim 7.27(\mathrm{~m}$, $3 \mathrm{H}), 7.22(\mathrm{~s}, 1 \mathrm{H}), 7.20(\mathrm{~d}, J=7.8 \mathrm{~Hz}, 1 \mathrm{H}), 7.11(\mathrm{~d}, J=7.8$ $\mathrm{Hz}, 1 \mathrm{H}), 6.31 \sim 6.29(\mathrm{~m}, 1 \mathrm{H}), 6.28(\mathrm{~d}, J=3.0 \mathrm{~Hz}, 1 \mathrm{H}), 5.19$ $(\mathrm{s}, 1 \mathrm{H}), 2.25(\mathrm{~s}, 3 \mathrm{H}), 2.23(\mathrm{~s}, 3 \mathrm{H}) ;{ }^{13} \mathrm{C}$ NMR $(150 \mathrm{MHz}$, $\left.\mathrm{CDCl}_{3}\right) \delta: 154.2,142.2,136.9,136.4,135.7,131.8,130.0$, 129.1, 128.3, 128.1, 125.3, 123.4, 110.3, 106.5, 87.8, 83.7, $37.6,19.9,19.4$; IR (KBr) v: 3054, 3016, 2922, 2860, 2362, 1603, 1595, 1497, 1448, $1176 \mathrm{~cm}^{-1}$; HRMS (ESI) calcd for $\mathrm{C}_{21} \mathrm{H}_{18} \mathrm{NaO}[\mathrm{M}+\mathrm{Na}]^{+}$309.1250, found 309.1258.

6-Chloro-3-(1-(3,4-dimethylphenyl)-3-phenylprop-2yn-1-yl)-1H-indole (2l): Yield $89 \%$, light yellow oil. ${ }^{1} \mathrm{H}$ NMR (600 MHz, $\left.\mathrm{CDCl}_{3}\right) \delta: 7.95(\mathrm{~s}, 1 \mathrm{H}), 7.51$ (d, $J=9.0$
$\mathrm{Hz}, 1 \mathrm{H}), 7.45 \sim 7.42(\mathrm{~m}, 2 \mathrm{H}), 7.30 \sim 7.24(\mathrm{~m}, 5 \mathrm{H}), 7.22(\mathrm{~d}$, $J=7.8 \mathrm{~Hz}, 1 \mathrm{H}), 7.10$ (d, $J=1.8 \mathrm{~Hz}, 1 \mathrm{H}), 7.08$ (d, $J=7.8$ $\mathrm{Hz}, 1 \mathrm{H}), 7.02$ (dd, $J=1.8,7.8 \mathrm{~Hz}, 1 \mathrm{H}), 5.34(\mathrm{~s}, 1 \mathrm{H}), 2.22$ (s, $6 \mathrm{H}) ;{ }^{13} \mathrm{C} \mathrm{NMR}\left(150 \mathrm{MHz}, \mathrm{CDCl}_{3}\right) \delta: 138.4,137.1,136.8$, $135.3,131.7,129.8,129.1,128.2,128.1,127.9,125.2$, $124.8,123.7,123.2,120.6,120.4,117.5,111.2,90.6,83.3$, $35.1,19.9,19.4$; IR (KBr) v: 3424, 3060, 3015, 2922, 2860, $1617,1495,1450,1334,1275,1130 \mathrm{~cm}^{-1}$; HRMS (ESI) calcd for $\mathrm{C}_{25} \mathrm{H}_{20} \mathrm{ClNNa}[\mathrm{M}+\mathrm{Na}]^{+}$392.1176, found 392.1181 .

3-(1-(3,4-Dimethylphenyl)-3-phenylprop-2-yn-1-yl)-6nitro-1 $H$-indole $(\mathbf{2 m})$ : Yield 95\%. Yellow solid, m.p. $>$ $170{ }^{\circ} \mathrm{C}$ (dec.); ${ }^{1} \mathrm{H}$ NMR $\left(600 \mathrm{MHz}, \mathrm{CDCl}_{3}\right) \delta: 8.52(\mathrm{~s}, 1 \mathrm{H})$, $8.32(\mathrm{~s}, 1 \mathrm{H}), 7.96(\mathrm{~d}, J=8.4 \mathrm{~Hz}, 1 \mathrm{H}), 7.67(\mathrm{~d}, J=9.0 \mathrm{~Hz}$, $1 \mathrm{H}), 7.50 \sim 7.40(\mathrm{~m}, 3 \mathrm{H}), 7.32 \sim 7.27(\mathrm{~m}, 3 \mathrm{H}), 7.25(\mathrm{~s}, 1 \mathrm{H})$, $7.22(\mathrm{~d}, J=7.8 \mathrm{~Hz}, 1 \mathrm{H}), 7.10(\mathrm{~d}, J=7.8 \mathrm{~Hz}, 1 \mathrm{H}), 5.40(\mathrm{~s}$, 1H), $2.24(\mathrm{~s}, 6 \mathrm{H}) ;{ }^{13} \mathrm{C}$ NMR $\left(150 \mathrm{MHz}, \mathrm{CDCl}_{3}\right) \delta: 143.5$, $137.8,137.0,135.6,135.2,131.7,130.8,130.0,129.0$, 128.3, 128.1, 125.1, 123.4, 119.7, 118.5, 115.2, 108.2, 89.9, 83.7, 34.9, 19.9, 19.4; IR (KBr) v: 3313, 3077, 2918, 1504, 1455, 1337, 1312, $1052 \mathrm{~cm}^{-1}$; HRMS (ESI) calcd for $\mathrm{C}_{25} \mathrm{H}_{21} \mathrm{~N}_{2} \mathrm{O}_{2}[\mathrm{M}+\mathrm{H}]^{+}$381.1598, found 381.1598.

4-(3-Phenyl-1-(3-(trifluoromethyl)phenyl)prop-2-yn-1-y 1)phenol (2n): Yield 82\%, yellow oil. ${ }^{1} \mathrm{H}$ NMR $(600 \mathrm{MHz}$, $\left.\mathrm{CDCl}_{3}\right) \delta: 7.70(\mathrm{~s}, 1 \mathrm{H}), 7.60(\mathrm{~d}, J=7.2 \mathrm{~Hz}, 1 \mathrm{H}), 7.50(\mathrm{~d}$, $J=7.8 \mathrm{~Hz}, 1 \mathrm{H}), 7.44 \sim 7.43(\mathrm{~m}, 2 \mathrm{H}), 7.43(\mathrm{t}, J=7.8 \mathrm{~Hz}$, $1 \mathrm{H}), 7.32 \sim 7.30(\mathrm{~m}, 3 \mathrm{H}), 7.28(\mathrm{~d}, J=8.4 \mathrm{~Hz}, 2 \mathrm{H}), 6.80(\mathrm{~d}$, $J=8.4 \mathrm{~Hz}, 2 \mathrm{H}), 5.20(\mathrm{~s}, 1 \mathrm{H}) ;{ }^{13} \mathrm{C} \mathrm{NMR}\left(150 \mathrm{MHz}, \mathrm{CDCl}_{3}\right)$ $\delta: 154.8,143.1,133.2,131.7,131.3,130.9\left(\mathrm{q}, J^{2}=32.6 \mathrm{~Hz}\right)$, $129.2,129.1,128.3,128.2,124.6\left(\mathrm{q}, J^{3}=3.3 \mathrm{~Hz}\right), 124.1(\mathrm{q}$, $\left.J^{1}=270.9 \mathrm{~Hz}\right), 123.8\left(\mathrm{q}, J^{3}=3.3 \mathrm{~Hz}\right), 123.2,115.7,89.4$, $85.5,42.8$; IR $(\mathrm{KBr}) v$ : 3371, 3061, 2931, 1605, 1511, 1444, 1330, 11238, 1167, 1126, $1074 \mathrm{~cm}^{-1}$; HRMS (ESI) calcd for $\mathrm{C}_{22} \mathrm{H}_{16} \mathrm{~F}_{3} \mathrm{O}[\mathrm{M}+\mathrm{H}]^{+}$353.1148, found 353.1147.

1-(1-(4-Methoxyphenyl)-3-phenylprop-2-yn-1-yl)-3(trifluoromethyl)benzene (2o): Yield $92 \%$, colorless oil. ${ }^{1} \mathrm{H}$ NMR $\left(600 \mathrm{MHz}, \mathrm{CDCl}_{3}\right) \delta: 7.71(\mathrm{~s}, 1 \mathrm{H}), 7.60$ (d, $J=7.2$ $\mathrm{Hz}, 1 \mathrm{H}), 7.50$ (d, $J=7.8 \mathrm{~Hz}, 1 \mathrm{H}), 7.48 \sim 7.46(\mathrm{~m}, 2 \mathrm{H}), 7.42$ $(\mathrm{t}, J=7.8 \mathrm{~Hz}, 1 \mathrm{H}), 7.33(\mathrm{~d}, J=8.4 \mathrm{~Hz}, 2 \mathrm{H}), 7.32 \sim 7.29(\mathrm{~m}$, $3 \mathrm{H}), 6.87(\mathrm{~d}, J=9.0 \mathrm{~Hz}, 2 \mathrm{H}), 5.21(\mathrm{~s}, 1 \mathrm{H}), 3.78(\mathrm{~s}, 1 \mathrm{H}) ;{ }^{13} \mathrm{C}$ NMR $\left(150 \mathrm{MHz}, \mathrm{CDCl}_{3}\right) \delta: 158.8,143.1,133.0,131.7$, $131.3,130.9\left(\mathrm{q}, J^{2}=32.6 \mathrm{~Hz}\right), 129.1,129.0,128.3,128.2$, $124.6\left(\mathrm{q}, J^{3}=3.2 \mathrm{~Hz}\right), 124.1\left(\mathrm{q}, J^{1}=270.9 \mathrm{~Hz}\right), 123.8(\mathrm{q}$, $\left.J^{3}=3.2 \mathrm{~Hz}\right), 123.2,114.2,89.5,85.4,55.3,42.8$; IR (KBr) $v: 2961,2840,2361,1601,1510,1447,1330,1256,1172$, $1126,1073 \mathrm{~cm}^{-1}$; HRMS (ESI) calcd for $\mathrm{C}_{23} \mathrm{H}_{18} \mathrm{~F}_{3} \mathrm{O}[\mathrm{M}+$ $\mathrm{H}]^{+}$367.1304, found 367.1305.

2-(3-Phenyl-1-(3-(trifluoromethyl)phenyl)prop-2-yn-1yl)furan (2p): Yield 80\%, light yellow oil. ${ }^{1} \mathrm{H}$ NMR $(600$ $\left.\mathrm{MHz} \mathrm{CDCl}_{3}\right) \delta: 7.75(\mathrm{~s}, 1 \mathrm{H}), 7.67(\mathrm{~d}, J=7.2 \mathrm{~Hz}, 1 \mathrm{H}), 7.54$ $(\mathrm{d}, J=7.8 \mathrm{~Hz}, 1 \mathrm{H}), 7.50 \sim 7.44(\mathrm{~m}, 3 \mathrm{H}), 7.35(\mathrm{~s}, 1 \mathrm{H})$, $7.33 \sim 7.30(\mathrm{~m}, 3 \mathrm{H}), 6.35 \sim 6.30(\mathrm{~m}, 2 \mathrm{H}), 5.31(\mathrm{~s}, 1 \mathrm{H}) ;{ }^{13} \mathrm{C}$ NMR $\left(150 \mathrm{MHz}, \mathrm{CDCl}_{3}\right) \delta: 152.8,142.6,140.0,131.8$, $131.3,131.2\left(\mathrm{q}, J^{2}=27.2 \mathrm{~Hz}\right), 129.2,128.5,128.4,128.2$, 124.7 (q, $\left.J^{3}=3.3 \mathrm{~Hz}\right), 124.4\left(\mathrm{q}, J^{3}=3.3 \mathrm{~Hz}\right), 124.1\left(\mathrm{q}, J^{1}=\right.$ $270.9 \mathrm{~Hz}), 122.8,110.5,107.1,86.4,84.7,37.7$; IR (KBr) $v$ : 3120, 3061, 2929, 1596, 1495, 1446, 1330, 1166, 1126, 
$1074 \mathrm{~cm}^{-1}$; HRMS (ESI) calcd for $\mathrm{C}_{20} \mathrm{H}_{14} \mathrm{~F}_{3} \mathrm{O}[\mathrm{M}+\mathrm{H}]$ 327.0991, found 327.0999.

4-(1-(4-Chlorophenyl)-3-phenylprop-2-yn-1-yl)phenol (2q): Yield 82\%, light yellow oil. ${ }^{1} \mathrm{H}$ NMR $(600 \mathrm{MHz}$, $\left.\mathrm{CDCl}_{3}\right) \delta: 7.48 \sim 7.44(\mathrm{~m}, 2 \mathrm{H}), 7.34(\mathrm{~d}, J=8.4 \mathrm{~Hz}, 2 \mathrm{H})$, $7.32 \sim 7.24(\mathrm{~m}, 7 \mathrm{H}), 6.78(\mathrm{~d}, J=9.0 \mathrm{~Hz}, 2 \mathrm{H}), 5.12(\mathrm{~s}, 1 \mathrm{H})$, $4.91(\mathrm{~s}, 1 \mathrm{H}) ;{ }^{13} \mathrm{C}$ NMR $\left(150 \mathrm{MHz}, \mathrm{CDCl}_{3}\right) \delta: 154.6,140.6$, $133.6,132.7,131.7,129.2,129.1,128.7,128.3,128.2$, 123.3, 115.6, 89.9, 85.1, 42.3; IR (KBr) v: 3357, 3056, 1599, 1510, 1489, 1442, 1234, 1173, $1093 \mathrm{~cm}^{-1}$;HRMS (ESI) calcd for $\mathrm{C}_{21} \mathrm{H}_{15} \mathrm{ClNaO}[\mathrm{M}+\mathrm{Na}]^{+} 341.0704$, found 341.0704

3-(1-(4-Chlorophenyl)-3-(4-methoxyphenyl)prop-2yn-1-yl)-6-nitro-1 $H$-indole (2r): Yield 88\%. Yellow solid, m.p. $>180{ }^{\circ} \mathrm{C}$ (dec.); ${ }^{1} \mathrm{H}$ NMR (600 MHz, $\left.\mathrm{CDCl}_{3}\right) \delta: 8.52$ $(\mathrm{s}, 1 \mathrm{H}), 8.34(\mathrm{~s}, 1 \mathrm{H}), 7.97(\mathrm{~d}, J=9.0 \mathrm{~Hz}, 1 \mathrm{H}), 7.62(\mathrm{~d}$, $J=7.8 \mathrm{~Hz}, 1 \mathrm{H}), 7.45 \sim 7.40(\mathrm{~m}, 3 \mathrm{H}), 7.37(\mathrm{~d}, J=7.8 \mathrm{~Hz}$, 2H), $7.31(\mathrm{~d}, J=7.8 \mathrm{~Hz}, 2 \mathrm{H}), 6.83$ (d, $J=7.8 \mathrm{~Hz}, 2 \mathrm{H}), 5.42$ $(\mathrm{s}, 1 \mathrm{H}), 3.81(\mathrm{~s}, 3 \mathrm{H}) ;{ }^{13} \mathrm{C}$ NMR $\left(150 \mathrm{MHz}, \mathrm{CDCl}_{3}\right) \delta: 159.6$, $143.7,139.2,135.3,133.1,133.0,130.5,129.2,128.9$, 128.2, 119.6, 118.0, 115.3, 115.1, 114.0, 108.2, 87.3, 84.2, 55.3, 34.8; IR (KBr) v: 3401, 1607, 1507, 1457, 1338, 1248, $1175 \mathrm{~cm}^{-1}$; HRMS (ESI) calcd for $\mathrm{C}_{24} \mathrm{H}_{18} \mathrm{ClN}_{2} \mathrm{O}_{3}$ $[\mathrm{M}+\mathrm{H}]^{+}$417.1000, found 417.1000.

3-(1-(4-(Benzyloxy)phenyl)oct-2-yn-1-yl)-6-nitro-1Hindole (2s): Yield 96\%. Yellow solid, m.p. 91 92 ${ }^{\circ} \mathrm{C} ;{ }^{1} \mathrm{H}$ NMR $\left(600 \mathrm{MHz}, \mathrm{CDCl}_{3}\right) \delta: 8.52(\mathrm{~s}, 1 \mathrm{H}), 8.30(\mathrm{~s}, 1 \mathrm{H}), 7.94$ $(\mathrm{d}, J=9.0 \mathrm{~Hz}, 1 \mathrm{H}), 7.58(\mathrm{~d}, J=8.4 \mathrm{~Hz}, 1 \mathrm{H}), 7.45 \sim 7.30(\mathrm{~m}$, $8 \mathrm{H}), 6.93(\mathrm{~d}, J=7.2 \mathrm{~Hz}, 2 \mathrm{H}), 5.17(\mathrm{~s}, 1 \mathrm{H}), 5.04(\mathrm{~s}, 2 \mathrm{H})$, $2.25(\mathrm{t}, J=6.6 \mathrm{~Hz}, 2 \mathrm{H}), 1.54(\mathrm{t}, J=6.6 \mathrm{~Hz}, 2 \mathrm{H}), 1.40 \sim 1.25$ $(\mathrm{m}, 4 \mathrm{H}), 0.88(\mathrm{t}, J=6.6 \mathrm{~Hz}, 3 \mathrm{H}) ;{ }^{13} \mathrm{C} \mathrm{NMR}(150 \mathrm{MHz}$, $\left.\mathrm{CDCl}_{3}\right) \delta: 157.8,143.4,137.0,135.2,133.6,130.7,128.8$, $128.6,128.1,128.0,127.5,119.8,119.3,115.0,114.9$, $108.1,84.1,80.2,70.1,34.0,31.2,28.6,22.2,18.9,14.0$; IR $(\mathrm{KBr}) v: 3344,2928,2857,1610,1508,1456,1323$, 1244, $1098 \mathrm{~cm}^{-1}$; HRMS (ESI) calcd for $\mathrm{C}_{29} \mathrm{H}_{29} \mathrm{~N}_{2} \mathrm{O}_{2}$ $[\mathrm{M}+\mathrm{H}]^{+}$453.2173, found 453.2173.

3-(1-(4-Chlorophenyl)oct-2-yn-1-yl)-6-nitro-1 $H$-indole (2t): Yield 85\%. Yellow solid, m.p. 98 100 ${ }^{\circ} \mathrm{C} ;{ }^{1} \mathrm{H}$ NMR $\left(600 \mathrm{MHz}, \mathrm{CDCl}_{3}\right) \delta: 8.55(\mathrm{~s}, 1 \mathrm{H}), 8.33(\mathrm{~s}, 1 \mathrm{H}), 7.95(\mathrm{~d}$, $J=9.0 \mathrm{~Hz}, 1 \mathrm{H}), 7.55(\mathrm{~d}, J=8.4 \mathrm{~Hz}, 1 \mathrm{H}), 7.38 \sim 7.34(\mathrm{~m}$, $3 \mathrm{H}), 7.28(\mathrm{~d}, J=7.8 \mathrm{~Hz}, 2 \mathrm{H}), 5.19(\mathrm{~s}, 1 \mathrm{H}), 2.26(\mathrm{t}, J=6.6$ $\mathrm{Hz}, 2 \mathrm{H}), 1.54(\mathrm{t}, J=6.6 \mathrm{~Hz}, 2 \mathrm{H}), 1.40 \sim 1.28(\mathrm{~m}, 4 \mathrm{H}), 0.88$ $(\mathrm{t}, J=6.6 \mathrm{~Hz}, 3 \mathrm{H}) ;{ }^{13} \mathrm{C} \mathrm{NMR}\left(150 \mathrm{MHz}, \mathrm{CDCl}_{3}\right) \delta: 143.4$, $139.8,135.3,132.8,130.6,129.1,128.7,128.4,119.6$, $118.4,115.1,108.4,84.8,79.5,34.2,31.1,28.6,22.2,18.8$, 14.0; IR (KBr) v: 3401, 2927, 2856, 1587, 1502, 1458, 1338, $1088 \mathrm{~cm}^{-1}$; HRMS (ESI) calcd for $\mathrm{C}_{22} \mathrm{H}_{22} \mathrm{ClN}_{2} \mathrm{O}_{2}$ $[\mathrm{M}+\mathrm{H}]^{+}$381.1364, found 381.1365.

1-(Benzyloxy)-4-(1-methoxyoct-2-yn-1-yl)benzene (2u): Yield 99\%, light yellow oil. ${ }^{1} \mathrm{H}$ NMR $\left(600 \mathrm{MHz}, \mathrm{CDCl}_{3}\right) \delta$ : $7.44 \sim 7.40(\mathrm{~m}, 4 \mathrm{H}), 7.38(\mathrm{t}, J=7.8 \mathrm{~Hz}, 2 \mathrm{H}), 7.32(\mathrm{t}, J=7.2$ $\mathrm{Hz}, 1 \mathrm{H}), 6.96(\mathrm{t}, J=7.8 \mathrm{~Hz}, 2 \mathrm{H}), 5.07(\mathrm{~s}, 2 \mathrm{H}), 5.02(\mathrm{~s}, 1 \mathrm{H})$, $3.37(\mathrm{~s}, 3 \mathrm{H}), 2.28(\mathrm{t}, J=6.6 \mathrm{~Hz}, 2 \mathrm{H}), 1.56(\mathrm{t}, J=7.2 \mathrm{~Hz}$, $2 \mathrm{H}), 1.41 \sim 1.25(\mathrm{~m}, 4 \mathrm{H}), 0.90(\mathrm{t}, J=6.6 \mathrm{~Hz}, 3 \mathrm{H}) ;{ }^{13} \mathrm{C} \mathrm{NMR}$ $\left(150 \mathrm{MHz}, \mathrm{CDCl}_{3}\right) \delta: 158.8,137.0,131.7,128.9,128.6$, $128.0,127.5,114.7,88.6,77.8,55.4,31.1,28.4,22.2,18.8$,
14.0; IR (KBr) v: 3064, 3034, 2932, 2864, 2819, 2271, 2219, 1610, 1509, 1460, 1380, 1243, $1079 \mathrm{~cm}^{-1}$; HRMS (ESI) calcd for $\mathrm{C}_{21} \mathrm{H}_{23} \mathrm{O}[\mathrm{M}-\mathrm{MeO}]^{+}$291.1743, found 291.1742.

1-(Benzyloxy)-4-(1-ethoxyoct-2-yn-1-yl)benzene (2v): Yield 99\%, colourless oil. ${ }^{1} \mathrm{H}$ NMR $\left(600 \mathrm{MHz} \mathrm{CDCl}_{3}\right) \delta$ : $7.44 \sim 7.40(\mathrm{~m}, 4 \mathrm{H}), 7.37(\mathrm{t}, J=7.2 \mathrm{~Hz}, 2 \mathrm{H}), 7.32(\mathrm{t}, J=6.6$ $\mathrm{Hz}, 1 \mathrm{H}), 6.95(\mathrm{~d}, J=6.6 \mathrm{~Hz}, 2 \mathrm{H}), 5.10(\mathrm{~s}, 1 \mathrm{H}), 5.06(\mathrm{~s}, 2 \mathrm{H})$, $3.70 \sim 3.60(\mathrm{~m}, 1 \mathrm{H}), 3.55 \sim 3.45(\mathrm{~m}, 1 \mathrm{H}), 2.26(\mathrm{t}, J=6.6$ $\mathrm{Hz}, 2 \mathrm{H}), 1.55(\mathrm{t}, J=6.0 \mathrm{~Hz}, 2 \mathrm{H}), 1.40 \sim 1.25(\mathrm{~m}, 4 \mathrm{H}), 1.22$ $(\mathrm{d}, J=2.4 \mathrm{~Hz}, 3 \mathrm{H}), 0.89$ (t, $J=6.6 \mathrm{~Hz}, 3 \mathrm{H}) ;{ }^{13} \mathrm{C}$ NMR $(150$ $\left.\mathrm{MHz}, \mathrm{CDCl}_{3}\right) \delta: 158.7,137.0,132.2,128.8,128.6,128.0$, $127.5,114.7,88.0,78.4,71.2,70.1,63.3,31.1,28.4,22.2$, $18.9,15.2,14.0$; IR (KBr) v: 3065, 3035, 2932, 2867, 1712, 1607, 1509, 1460, 1382, 1247, $1107 \mathrm{~cm}^{-1}$; HRMS (ESI) calcd for $\mathrm{C}_{23} \mathrm{H}_{28} \mathrm{NaO}_{2}[\mathrm{M}+\mathrm{Na}]^{+}$359.1982, found 359.1982 .

1-(Benzyloxy)-4-(1-isopropoxyoct-2-yn-1-yl)benzene (2w): Yield 99\%, light yellow oil. ${ }^{1} \mathrm{H}$ NMR $(600 \mathrm{MHz}$, $\left.\mathrm{CDCl}_{3}\right) \delta: 7.44 \sim 7.40(\mathrm{~m}, 4 \mathrm{H}), 7.37(\mathrm{t}, J=6.6 \mathrm{~Hz}, 2 \mathrm{H}), 7.31$ $(\mathrm{t}, J=6.6 \mathrm{~Hz}, 1 \mathrm{H}), 6.95(\mathrm{~d}, J=6.6 \mathrm{~Hz}, 2 \mathrm{H}), 5.14(\mathrm{~s}, 1 \mathrm{H})$, $5.06(\mathrm{~s}, 2 \mathrm{H}), 3.97 \sim 3.90(\mathrm{~m}, 1 \mathrm{H}), 2.25(\mathrm{t}, J=6.6 \mathrm{~Hz}, 2 \mathrm{H})$, $1.53(\mathrm{t}, J=6.0 \mathrm{~Hz}, 2 \mathrm{H}), 1.40 \sim 1.25(\mathrm{~m}, 4 \mathrm{H}), 1.22(\mathrm{~d}, J=3.6$ $\mathrm{Hz}, 3 \mathrm{H}), 1.19$ (d, $J=2.4 \mathrm{~Hz}, 3 \mathrm{H}), 0.89$ (t, $J=6.6 \mathrm{~Hz}, 3 \mathrm{H})$; ${ }^{13} \mathrm{C}$ NMR $\left(150 \mathrm{MHz}, \mathrm{CDCl}_{3}\right) \delta: 158.6,137.1,132.8,128.7$, 128.6, 127.9, 127.4, 114.7, 87.3, 79.0, 70.1, 69.1, 68.7, 31.1, 28.4, 22.9, 22.2, 21.8, 18.9, 14.0; IR (KBr) v: 3065, 3035, 2932, 2867, 1710, 1607, 1509, 1461, 1378, 1247, 1172, $1104,1027 \mathrm{~cm}^{-1}$; HRMS (ESI) calcd for $\mathrm{C}_{24} \mathrm{H}_{30} \mathrm{NaO}_{2}[\mathrm{M}+$ $\mathrm{Na}]^{+}$373.2138, found 373.2139.

1-(Benzyloxy)-4-(1-(cinnamyloxy)oct-2-yn-1-yl)benzene (2x): Yield 99\%, colourless oil. ${ }^{1} \mathrm{H}$ NMR $(600 \mathrm{MHz}$, $\left.\mathrm{CDCl}_{3}\right) \delta: 7.45(\mathrm{~d}, J=7.8 \mathrm{~Hz}, 2 \mathrm{H}), 7.42(\mathrm{~d}, J=6.6 \mathrm{~Hz}, 2 \mathrm{H})$, $7.40 \sim 7.35(\mathrm{~m}, 4 \mathrm{H}), 7.34 \sim 7.28(\mathrm{~m}, 3 \mathrm{H}), 7.26 \sim 7.20(\mathrm{~m}$, $1 \mathrm{H}), 6.97(\mathrm{~d}, J=7.8 \mathrm{~Hz}, 2 \mathrm{H}), 6.62(\mathrm{~d}, J=16.2 \mathrm{~Hz}, 1 \mathrm{H})$, $6.35 \sim 6.28(\mathrm{~m}, 1 \mathrm{H}), 5.20(\mathrm{~s}, 1 \mathrm{H}), 5.07(\mathrm{~s}, 2 \mathrm{H}), 4.32 \sim 4.20$ $(\mathrm{m}, 2 \mathrm{H}), 2.29(\mathrm{t}, J=6.6 \mathrm{~Hz}, 2 \mathrm{H}), 1.59 \sim 1.53(\mathrm{~m}, 2 \mathrm{H})$, $1.43 \sim 1.25(\mathrm{~m}, 4 \mathrm{H}), 0.90(\mathrm{t}, J=6.6 \mathrm{~Hz}, 3 \mathrm{H}) ;{ }^{13} \mathrm{C} \mathrm{NMR}(150$ $\left.\mathrm{MHz}, \mathrm{CDCl}_{3}\right) \delta: 158.8,137.0,136.8,132.8,131.9,129.0$, $128.6,128.5,128.0,127.7,127.5,126.5,126.0,114.8,88.5$, $78.1,70.5,70.1,68.4,31.1,28.4,22.2,18.9,14.0$; IR (KBr) $v: 3031,2931,1608,1509,1457,1380,1241,1043 \mathrm{~cm}^{-1}$; HRMS (ESI) calcd for $\mathrm{C}_{30} \mathrm{H}_{32} \mathrm{NaO}_{2}[\mathrm{M}+\mathrm{Na}]^{+}$447.2295, found 447.2293.

1-Chloro-4-(1-(cinnamyloxy)oct-2-yn-1-yl)benzene (2y): Yield 85\%, colourless oil. ${ }^{1} \mathrm{H}$ NMR $(600 \mathrm{MHz}$, $\left.\mathrm{CDCl}_{3}\right) \delta: 7.47(\mathrm{~d}, J=6.6 \mathrm{~Hz}, 2 \mathrm{H}), 7.38(\mathrm{~d}, J=7.8 \mathrm{~Hz}, 2 \mathrm{H})$, $7.36 \sim 7.29(\mathrm{~m}, 4 \mathrm{H}), 7.26 \sim 7.22(\mathrm{~m}, 1 \mathrm{H}), 6.63(\mathrm{~d}, J=15.6$ $\mathrm{Hz}, 1 \mathrm{H}), 6.35 \sim 6.28(\mathrm{~m}, 1 \mathrm{H}), 5.22(\mathrm{~s}, 1 \mathrm{H}), 5.07(\mathrm{~s}, 2 \mathrm{H})$, $4.35 \sim 4.22(\mathrm{~m}, 2 \mathrm{H}), 2.29(\mathrm{t}, J=6.6 \mathrm{~Hz}, 2 \mathrm{H}), 1.60 \sim 1.52$ $(\mathrm{m}, 2 \mathrm{H}), 1.42 \sim 1.25(\mathrm{~m}, 4 \mathrm{H}), 0.91(\mathrm{t}, J=6.0 \mathrm{~Hz}, 3 \mathrm{H}) ;{ }^{13} \mathrm{C}$ NMR $\left(150 \mathrm{MHz}, \mathrm{CDCl}_{3}\right) \delta: 137.9,136.7,134.0,133.1$, $128.9,128.6,127.8,126.5,125.6,89.1,77.5,70.2,68.6$, $31.1,28.3,22.2,18.8,14.0$; IR (KBr) v: 3028, 2956, 2930, 2857, 1647, 1588, 1489, 1459, 1262, $1090 \mathrm{~cm}^{-1}$; HRMS (ESI) calcd for $\mathrm{C}_{23} \mathrm{H}_{25} \mathrm{ClNaO}[\mathrm{M}+\mathrm{Na}]^{+} 375.1486$, found 375.1485 . 
Supporting Information Typical procedure for the preparation of 1,3-disubstituted propargyl alcohols $1 ;{ }^{1} \mathrm{H}$ NMR and ${ }^{13} \mathrm{C}$ NMR spectra of $\mathbf{1 a} \sim \mathbf{1 h} ;{ }^{1} \mathrm{H}$ NMR and ${ }^{13} \mathrm{C}$ NMR spectra of $\mathbf{2} \mathbf{a} \sim \mathbf{2 y}$. The Supporting Information is available free of charge via the Internet at http://siocjournal.cn/.

\section{References}

[1] (a) Zhu, H.; Ji, K.; Yang, F.; Wang, L.; Zhao, S.; Ali, S.; Liu, X.; Liang, Y. Org. Lett. 2011, 13, 684.

(b) Roy R.; Saha S. RSC Adv. 2018, 8, 31129.

(c) Zhang, X. X.; Lü, C.; Li, P.; Fu, B.; Yao, W. W. Chin. J. Org. Chem. 2016, 36, 1287 (in Chinese).

(张小祥, 吕昌, 李萍, 付博, 姚薇薇, 有机化学, 2016, 36, 1287.)

[2] (a) Georgy, M.; Boucard, V.; Campagne, J. J. Am. Chem. Soc. 2005, 127, 14180 .

(b) Trost, B. Acc. Chem. Res. 2002, 35, 695.

(c) Georgy, M.; Boucard, V.; Campagne, J. J. Am. Chem. Soc. 2005, 127, 14180 .

(d) Emer, E.; Sinisi, R.; Capdevila, M. G.; Petruzziello, D.; Vincentiis, F. D.; Cozzi, P. G. Eur. J. Org. Chem. 2011, 647.

[3] (a) Nicholas, K. M.; Mulvaney, M.; Bayer, M. J. Am. Chem. Soc. 1980, 102, 2508.

(b) Nicholas, K. M. Acc. Chem. Res. 1987, 20, 207.

(c) Berge, J.; Claridge, S.; Mann, A.; Muller, C.; Tyrrell, E. Tetrahedron Lett. 1997, 38, 685.

(d) Kuhn, O.; Rau, D.; Mayr, H. J. Am. Chem. Soc. 1998, 120, 900.

(e) Montana, A. M.; Fernandez, D. Tetrahedron Lett. 1999, 40, 6499.

(f) Teobald, B. J. Tetrahedron 2002, 58, 4133.

[4] (a) Sherry, B. D.; Radosevich, A. T.; Toste, F. D. J. Am. Chem. Soc. 2003, 125, 15760 .

(b) Kennedy-Smith, J. J.; Young, L. A.; Toste, F. D. Org. Lett. 2004, 6,1325 .

(c) Ohri, R. V.; Radosevich, A. T.; Hrovat, K. J.; Musich, C.; Huang, D.; Holman, T. R. Org. Lett. 2005, 7, 2501.

[5] (a) Nishibayashi, Y.; Wakiji, I.; Hidai, M. J. Am. Chem. Soc. 2000, 122,11019 .

(b) Nishibayashi, Y.; Wakiji, I.; Ishii, Y.; Uemura, S.; Hidai, M. J. Am. Chem. Soc. 2001, 123, 3393.

(c) Nishibayashi, Y.; Yoshikawa, M.; Inada, Y.; Hidai, M.; Uemura, S. J. Am. Chem. Soc. 2002, 124, 11846.

(d) Inada, Y.; Nishibayashi, Y.; Hidai, M.; Uemura, S. J. Am. Chem. Soc. 2002, 124, 15172.

(e) Nishibayashi, Y.; Yoshikawa, M.; Inada, Y.; Hidai, M.; Uemura, S. J. Am. Chem. Soc. 2002, 14, 11846.

(f) Nishibayashi, Y.; Inada, Y.; Hidai, M.; Uemura, S. J. Am. Chem. Soc. 2003, 125, 6060 .

(g) Nishibayashi, Y.; Yoshikawa, M.; Inada, Y.; Hidai, M.; Uemura, S. Angew. Chem., Int. Ed. 2003, 42, 1495.

(h) Cadierno, V.; Diez, J.; Garcia-Garrido, S. E.; Gimerno, J. Chem. Commun. 2004, 2716.

(i) Fischmeister, C.; Toupet, L.; Dixneuf, P. H. New J. Chem. 2005,
$29,765$.

(j) Nishibayashi, Y.; Milton, M. D.; Inada, Y.; Yoshikawa, M.; Wakiji, I.; Hidai, M.; Uemura, S. Chem.-Eur. J. 2005, 11, 1433.

(k) Nishibayashi, Y.; Uemura, S. Curr. Org. Chem. 2006, 10, 135.

(1) Bustelo, E.; Dixneuf, P. H. Adv. Synth. Catal. 2007, 349, 933.

[6] (a) Zhan, Z.; Yang, W.; Yang, R.; Yu, J.; Li, J.; Liu, H. Chem. Commun. 2006, 3352.

(b) Jana, U.; Maiti, S.; Biswas, S. Tetrahedron Lett. 2007, 48, 7160. (c) Yan, W.; Wang, Q.; Chen, Y.; Petersen, J. L.; Shi, X. Org. Lett. 2010, 12, 3308.

(d) Mantione, R. Bull. Soc. Chim. Fr. 1969, 4514

(e) Hayashi, M.; Inbushi, A.; Mukaiyama, T. Bull. Soc. Chem. Jpn. 1988, 61, 4037.

(f) Shi, M.; Shouki, K.; Okamoto, Y.; Takamuku, S. J. Chem. Soc., Perkin Trans. 1 1990, 2443.

(g) Burgess, K.; Jennings, L. D. J. Am. Chem. Soc. 1991, 6129.

[7] (a) Zhang, X.; Teo, W.; Chan, W. Org. Lett. 2009, 11, 4990.

(b) Gohain, M.; Marais, C.; Bezuidenhoudt, B. C. Tetrahedron Lett. 2012, 53, 1048 .

[8] (a) Sanz, R.; Martínez, A.; Álvarez-Gutiérrez, J.; Rodríguez, F. Eur J. Org. Chem. 2006, 1383.

(b) Sanz, R.; Miguel, D.; Martínez, A.; Álvarez-Gutiérrez, J.; Rodríguez, F. Org. Lett. 2007, 9, 727.

(c) Sanz, R.; Miguel, D.; Martínez, A.; Gohain, M.; García-García, P.; Fernández-Rodríguez, M.; Álvarez, E.; Rodríguez, F. Eur. J. Org. Chem. 2010, 7027.

(d) Gangadhararao, G.; Uruvakilli, A.; Kumara Swamy, K. C. Org. Lett. 2014, 16, 6060.

(e) Zhang, X.; Teng, W.; Sally, T.; Wai, P.; Chan, H. J. Org. Chem. 2010, 75, 6290 .

(f) Wang T.; Chen X.; Chen L.; Zhan Z. Org. Lett. 2011, 13, 3324.

[9] Barreiro, E.; Vidal, A. S.; Tan, E.; Lau, S.; Sheppard, T. D.; González, S. D. Eur. J. Org. Chem. 2015, 7544.

[10] Savarimuthu, S. A.; Prakash, D. L.; Thomas, S. A. Tetrahedron Lett. 2014, 55, 3213.

[11] Yokosaka, T.; Shiga, N.; Nemoto, T.; Hamada, Y. J. Org. Chem. 2014, 79, 3866.

[12] Wang, L.; Xie, X.; Liu, Y. Org. Lett. 2012, 23, 5848.

[13] (a) Shaterian, H. R.; Yarahmadi, H.; Ghashang, M. Tetrahedron 2008, 64, 1263.

(b) Murugan, K.; Chen, C. Tetrahedron Lett. 2011, 52, 5827.

[14] (a) Yadav, J. S.; Reddy, B. V. S.; Reddy, A. S. J. Mol. Catal. A: Chem. 2008, 280, 219.

(b) Srihari, P.; Reddy, J. S. S.; Bhunia, D. C.; Mandal, S. S.; Yadav, J. S. Synth. Commun. 2008, 38, 1448.

(c) Srihari, P.; Reddy, J. S. S.; Mandal, S. S.; Satyanarayana, K.; Yadav, J. S. Synthesis 2008, 1853.

(d) Jang, S.; Kim, A. Y.; Seo, W. S.; Park, K. H. Nanoscale Res. Lett. 2015, 10, 2.

[15] Antonino, J. R. C.; Prez, A. L.; Corma, A. Angew. Chem., Int. Ed. 2015, 54, 5658.

[16] Yadav, J. S.; Reddy, B. V. S.; Narayanakumar, G. G. K. S.; Rao, K. V. R. Chem. Lett. 2007, 36, 942.

[17] Gujarathi, S.; Hendrickson, H. P.; Zheng, G. Tetrahedron Lett. 2013, 54,3550 .

(Lu, Y.) 